Research article

\title{
Two-dimensional inversion modeling of magnetotelluric (MT) synthetic data of a graben structure using SimPEG
}

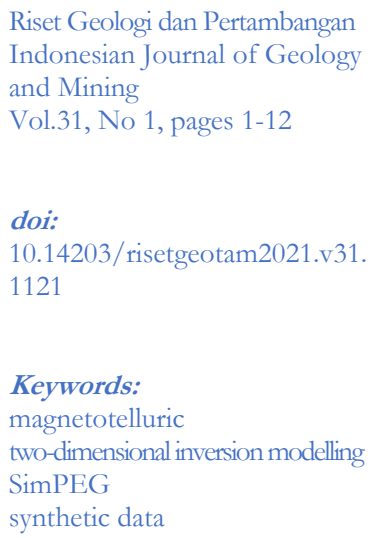

(C)2021 Pusat Penelitian Geoteknologi - Lembaga Ilmu Pengetahuan Indonesia

This is an open access article under the CC BY-NC-ND license

(http://creativecommons.org/1 icenses/by-nc-nd/4.0/).

\section{Imamal Muttaqien*, Jajang Nurjaman}

Department of Physic, Faculty of Science and Technology, Sunan Gunung Djati Islamic National University, Bandung

\begin{abstract}
The magnetotelluric (MT) method is a passive exploration method in geophysics that utilizes natural electromagnetic waves as a signal source. MT operates in the frequency range of 10-5 - $106 \mathrm{hertz}$, designed to study the structure of the conductivity below the earth's surface with a depth range from several tens of meters to the upper mantle. In this paper, 2-dimensional inversion modeling is performed on MT synthetic data using the SimPEG software. First, forward modeling is done by making a 2-dimensional conductivity model in the form of a valley (graben), which aims to produce MT synthetic data in TE (transverse electric) mode, TM (transverse magnetic) mode, and a combination of TE mode and TM mode. Next, an inversion modeling is performed on the MT synthetic data by adding a 5\% Gaussian noise and a 10-5 floor as data uncertainty to obtain a 2-dimensional conductivity inversion model. The final result can be validated by comparing the true model and the inversion model and between observational data (synthetic) and predictive predicted data. The results of this study provide a significant fit of the model and suitability of the data. The inversion quality is validated with an RMS Error for TE mode of $0.349 \%$, TM mode of $0.348 \%$, and a combination of TE and TM mode of $0.249 \%$.
\end{abstract}

\section{INTRODUCTION}

Magnetotelluric (MT) is one of the geophysical methods used to obtain information about the physical conditions below the earth's surface. First introduced by Tikhonov (1950), Rikitake (1948), and Cagniard (1953), the MT method is applied to study the structure of the earth's conductivity. One of the required steps to obtain the subsurface model is forward and inversion modeling.

Previously, geophysical modeling used free software written in Fortran and C/C++. Those modeling programs require other software to visualize the data, such as MARE2DEM (Key, 2016) and RES2DINV (Loke and Barker, 1996). In the last five years, the paradigm of geophysicists in various parts of the world has shifted along with the growing popularity of Python. Python is multipurpose software. In addition to performing calculations, this software can achieve data visualization. Some geophysical data modeling software written in Python and accessible for free include SimPEG (Cockett et al., 2015), pyGIMLi (Rücker et al., 2017), Fatiando (Uieda et al., 2013), GemPy (Varga et al., 2019) ) and Pyrocko 
(Heimann et al., 2019). The software developers also provide discussion forums such as the Google Group and Slack that help software users solve various problems related to the software.

SimPEG (Simulation and Parameter Estimation in Geophysics) is a Python package for simulating and estimating geophysical parameters, based on the finite volume method for forward modeling and the gradient method for inversion modeling. SimPEG is used to describe images of structures below the earth's surface in 1, 2, and 3 dimensions (Cockett et al., 2015). Several other geophysical methods that use SimPEG for data modeling are geoelectric, gravity, magnetic, induced polarization (IP), frequencydomain electromagnetic (FDEM), and time-domain electromagnetic (TDEM).

\section{METHODS}

\section{Magnetotelluric (MT)}

Magnetotelluric is a passive geophysical method, which measures natural sources of electromagnetic (EM) waves (Unsworth, 2007). MT data obtained from the simultaneous measurement of the total EM field with respect to time variations. The MT method measures electric and magnetic fields in orthogonal directions at the earth's surface (Simpson and Bahr, 2005), utilizing the electric and natural magnetic fields, which surround the earth (Sism and Bostick, 1969). The MT method is used to understand the structural conductivity of the earth at depths from several tens of meters to the upper mantle (Vozoff and Nabighian, 1991). This understanding is obtained by estimating the distribution pattern of the subsurface conductivity through the differences in the recorded signal.

The MT method measures frequencies in a range of $10^{-5}-10^{6} \mathrm{~Hz}$ (Chase and Jones, 2012). At frequencies below $1 \mathrm{~Hz}$, most of the MT signal sources come from systems in the magnetosphere formed by solar activity. For the frequency range between $1-10^{4} \mathrm{~Hz}$, the source of the MT signal comes from lightning storms around the world which emit plane waves and then spread and move around the earth (Vozoff and Nabighian, 1991). As for frequencies between $10-1000 \mathrm{kHz}$, the source of the MT signal comes from a radio transmitter or called radio-magnetotelluric (RMT) (Tezkan et al., 2018).

MT is based on the induction of EM waves by electric currents below the earth's surface (Bücker et al., 2017). The principle of EM wave propagation starts from a magnetic field source that reaches the earth's surface, some will be reflected, and some will penetrate deeper. Magnetic induction, which produces an electric current (Eddie current), occurs when the primary magnetic field propagates in the earth because the earth is a good conductor.

Electric current will interact in the conducting medium and produce a secondary magnetic field (Simpson and Bahr, 2005). This change in the secondary magnetic field over time provides information on the size and location of the conducting medium (Unsworth, 2009).

The Maxwell equation is an empirical equation that describes the behavior of the EM field (Zonge et al., 1991).

$$
\vec{\nabla} \times \vec{E}=-\frac{\partial \vec{B}}{\partial t}
$$

where $\vec{E}$ is the electric field (volt/meter), $\vec{B}$ is the flux or magnetic induction (Tesla) and $t$ is time (seconds). Equation (1) is Faraday's law, which states that a change in magnetic flux will cause an electric field, with an electromotive force in the opposite direction to the variation in the magnetic flux that causes it.

$$
\vec{\nabla} \times \vec{H}=\vec{J}+\frac{\partial \vec{D}}{\partial t}
$$


where $\vec{H}$ is the magnetic field (ampere/meter), $\vec{J}$ is the electric current flux (ampere/meter ${ }^{2}$ ) and $\vec{D}$ is the displacement current (coulomb/meter ${ }^{2}$ ). Equation (2) is a generalization of the Ampere-Maxwell equation, which states that the magnetic field arises as a result of the total flux of electric current caused by conduction currents and displacement currents.

A wave equation can be derived from Maxwell's equation, which can then be reduced to a diffusion equation employing a quasi-stationary approximation. This quasi-stationary approximation This quasistationary approximation states that the displacement current is negligible relative to the conductivity current in the period range of 10-5 - $106 \mathrm{~s}$, with resistivity values $<10000 \mathrm{~m}$ and negligible travel time of EM waves. Thus, the propagation of the EM field through the earth can be explained as a diffusive process (Naidu, 2012), as follows:

$$
\begin{aligned}
& \nabla^{2} E=k^{2} E \\
& \nabla^{2} H=k^{2} H
\end{aligned}
$$

where $\mathrm{k}$ is the wavenumber, whose magnitude is:

$$
k=i \omega \mu_{0} \sigma
$$

where $i$ is an imaginary number, $\omega$ is the angular frequency of the equation $(\omega=2 \pi f)$ with frequency $(f)$ in hertz, $\mu_{0}$ magnetic permeability $\left(\mu_{0}=\mu=4 \pi \times 10^{-7}\right.$ Henry/meter $)$ and $\sigma$ is conductivity (Siemens/meter).

EM waves are considered to propagate vertically downwards so that the electric field measurements are mutually perpendicular to the magnetic field (Naidu, 2012). Information on subsurface conductivity distribution can be obtained by comparing the intensity between the electric field (as the output) and the magnetic field (as the input), called the impedance, which is a complex number.

$$
Z=\frac{E}{H}=\left[\begin{array}{ll}
Z_{x x} & Z_{x y} \\
Z_{y x} & Z_{y y}
\end{array}\right]
$$

From this complex impedance, we can obtain the apparent resistivity (in Ohm meter):

$$
\rho_{a}=\frac{1}{\omega \mu_{0}}|Z|^{2}
$$

and phase (in degrees):

$$
\phi=\tan ^{-1}\left|\frac{\operatorname{ImZ}}{\operatorname{ReZ}}\right|
$$

From the solution of electric and magnetic fields, the amplitude of the EM wave will be attenuated (Chave and Jones, 2012). The parameter used to estimate the penetration depth of EM waves due to attenuation is skin depth. Here, the EM field is attenuated to $\mathrm{e}^{-1}$, from an amplitude at the earth's surface, which is conductive with an attenuation rate dependent on the conductivity of the medium through which it penetrates (Simpson and Bahr, 2005).

$$
\delta=\sqrt{\frac{2 \rho}{\omega \mu_{0}}}=503 \sqrt{\rho T}
$$


where $\delta$ is the skin depth (meter), $\rho$ is resistivity (Ohmmeter), and Tis period (seconds).

\section{Two-Dimensional Earth Model}

A simple description of the 2-dimensional earth model is the vertical contact between 2 different conductivity zones (Simpson and Bahr, 2005). The medium is characterized by the direction of the structure (strike) along the conductive structure, and the source of the EM field is constant (Chave and Jones, 2012). The conductivity is constant along one horizontal direction but changes along the other vertical and horizontal directions (Naidu, 2012).

In the 2-dimensional case, the electric and magnetic fields are perpendicular to each other, so there are two modes in MT, namely TE mode and TM mode. The mode is obtained as a consequence of the boundary conditions in the EM field.

TE mode (Transverse Electric) or E-polarization is a condition when the electric field is parallel to the strike direction (Simpson and Bahr, 2005), which causes the magnetic field to vary in the direction perpendicular to the strike and the vertical direction of the bedding plane. While the TM mode (Transverse Magnetic) or H-polarization is a condition when the magnetic field is parallel to the strike direction (Simpson and Bahr, 2005), which causes the electric field to vary in the direction perpendicular to the strike and the vertical direction of the bedding plane.

In this paper, SimPEG applies a coordinate system, namely the positive x-axis to the East and the y-axis to the North in the horizontal direction, and the z-axis in the vertical direction. Here, the strike direction is parallel to the $y$-axis so that the TE mode is the yx polarization and the TM mode is the xy polarization.

\section{Model Parameter Initialization}

The earth model parameters used in this study are shown in Table 1.

Table 1. The earth model parameters.

\begin{tabular}{ll}
\hline Name & Constant (S/m) \\
\hline Air Conductivity & $1-10^{-8}$ \\
Sediment Conductivity & $1-10^{-3}$ \\
Surface Conductivity & $1-10^{-2}$ \\
Depth Conductivity & $1-10^{-1}$ \\
Number of Frequencies & 20 \\
\hline
\end{tabular}

\section{Discretization}

Any application of a numerical method requires discretization from a continuous function into a discrete approximation. The main problem in the discretization process is to design the mesh that provides accurate solutions.

There are three main parameters in mesh discretization, namely the number of mesh in the horizontal and vertical directions, as well as the highest and lowest conductivity and frequency values. These parameters can be obtained by calculating the minimum and maximum skin depth using Equation (9). It aims to determine the depth of the wave propagating through the medium, which is considered homogeneous based on the given conductivity and frequency function. 
The results can be used to determine the smallest cell size for the vertical direction and maximum depth extension in constructing core domains which generally have higher density levels given by the following equation:

$$
\mathrm{Cs}_{z}=\frac{\delta_{\min }}{2}
$$

where $\mathrm{cs}_{\mathrm{z}}$ is the smallest cell size for the vertical direction and $\delta_{\min }$ is the minimum skin depth.

Equation (10) determines the smallest cell size for the vertical direction with minimum skin depth. In contrast, the cell size for the horizontal direction(css) is half of the distance between stations. The extension domain is determined based on the maximum skin depth for all receivers, as follows:

$$
\text { extent }=2 \times \delta_{\max }
$$

where $\delta_{\max }$ is the maximum skin depth.

\section{Forward Modelling}

In this study, a real graben structure was chosen as the conceptual model. Graben is a depression morphology formed by normal faults on one or both sides. This model describes the subsurface structure with an active layer smaller than zero (z-axis). Information regarding the survey includes five measurement stations, the receivers are located at positions $-12,-6,0,6$, and 12 (in $\mathrm{km}$ ) on the $\mathrm{x}$-axis, the distance between stations is $6 \mathrm{~km}$, with source polarization TE mode, TM mode, and a combination of TE mode and TM mode. Furthermore, the true model is referred to as the review area.

The graben-shaped model and information about the MT data acquisition were chosen, referring to the ultimate goal of this research. SimPEG software will be applied to the MT data from an area with basin morphology. The distance between measurement stations and the number of measurement stations are adjusted to this morphological dimension.

Figure 1 shows the true model with a width of $30 \mathrm{~km}$ and a depth of $4 \mathrm{~km}$, with a conductivity value of $10^{-1} \mathrm{~S} / \mathrm{m}$ as environmental conductivity and a conductivity value of $10^{-3} \mathrm{~S} / \mathrm{m}$ as sediment conductivity.

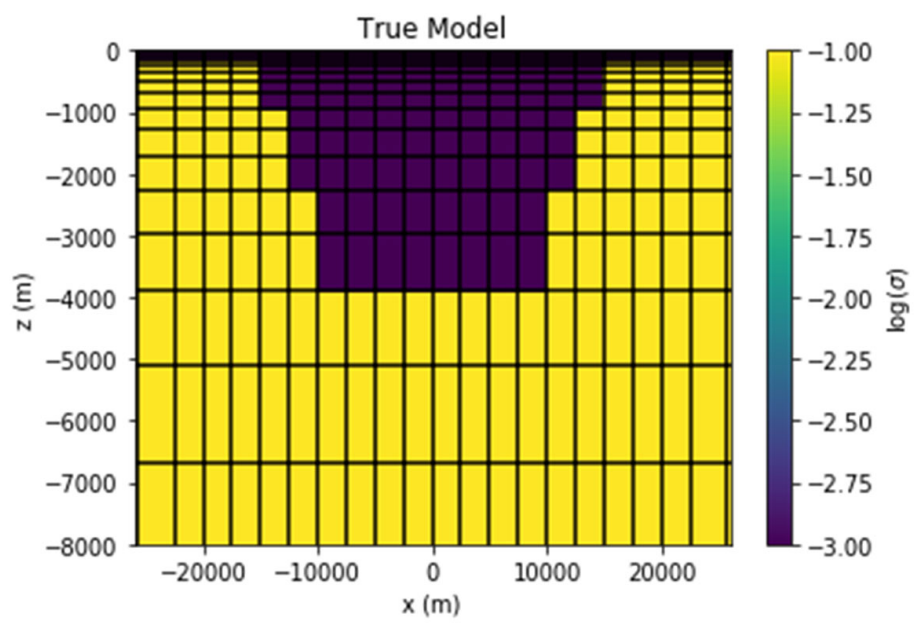

Figure 1. True model (graben). 
In MT, synthetic data contains complex impedance data based on the number of frequencies, the number of measurement stations, and the number of polarization sources. Furthermore, the synthetic data is treated as observational data by adding Gaussian noise by estimating the standard deviation for each datum and a random number (floor) with positive or negative values around zero as data uncertainty normally distributed:

$$
\varepsilon_{i}=\%\left|d_{i}\right|+\text { floor }
$$

where $\varepsilon_{i}$ is the data uncertainty in each datum, $d_{i}$ is the datum, \% is the standard deviation, and floor is a random number.

In this study, synthetic MT data was added with 5\% Gaussian noise and a floor of $10^{-5}$ as data uncertainty. Furthermore, the calculation of apparent resistivity and phase for each data measurement station is carried out using Equation (7) and Equation (8).

\section{Inversion}

In geophysical modeling, inversion is performed to estimate model parameters that provide predictive data (calculations) as a model response, according to observational data (Grandis, 2009). Most of the inversion problems in geophysics are non-linear, namely, connecting the data with model parameters. In general, it can be stated as:

$$
d=G(m)+e
$$

where $d$ is the observation data, $m$ is the model parameter, $G$ is the relationship between $d$ and $m$ or commonly referred to as forward modeling, and $e$ is the error data vector from $d$.

In SimPEG, two important elements of the inversion problem are formulated mathematically (Cockett et al., 2015). First, data misfit is the discrepancy between the observed data and the predicted data. Second, data discrepancies can be considered disturbances, errors in the description of survey parameters, and others.

For conformity assessment, the L2 norm is used as a measure or distance of misfit in the least square, which indicates the distance between the observed data and the calculated data. The objective function of the data misfit is:

$$
\phi_{d}=(d-G(m))^{T} W(d-G(m))
$$

where $\phi$ is the objective function for the misfit data and $W$ is the covariance matrix of the error data vector formed by $(W=1 / \varepsilon i)$ from Equation (12).

The most commonly used approach to solving non-linear inversion problems is the linearization approach with a first-order Taylor series expansion of the G(m) function around the initial model (m0), ignoring higher orders (Grandis, 2009). The solution to obtain the optimum model is through an iterative process of perturbation of the model to the initial model.

$$
m_{n+1}=m_{n}+\left[J_{n}{ }^{T} J_{n}\right]^{T} J_{n}{ }^{T}(d-G(m))
$$

where $\mathrm{J}$ is the Jacobian matrix. It is commonly referred to as the sensitivity matrix in the form of a partial derivative of the function $\mathrm{G}(\mathrm{m})$ with respect to the model parameters $\mathrm{m}$. 
Equation (15) is an optimization equation that expresses as a non-linear inversion solution performed with a linear approach known as the Gauss-Newton method used to update the model.

Second, regularization is used to evaluate the model with the desired characteristics and in accordance with previously known information. Therefore, the application of the inversion problem is extended by using the objective function of the model, as follows:

$$
\phi_{m}=\int_{V}|L m|^{2} d V=m^{T} L^{T} V L m
$$

where $\phi_{m}$ is the objective function for the model, $L$ is the model covariance matrix as the operator of the function $\left(|L m|^{2}=\int \nabla\left(m-m_{0}\right)^{2} d V\right.$, so that $L m$ approaches the Laplacian of log $\rho$ and $V$ is the domain for the model function with a value of 1 .

A realistic structural model is obtained by minimizing Equation (14) and Equation (16). The following is a general solution to the inversion problem, which is an objective function as the relationship between the data misfit function and the model function, as follows:

$$
\psi=\phi_{d}+\beta \phi_{m}
$$

where $\psi$ is the objective function, and $\beta$ is the regularization parameter that has a positive value ( $\beta$ $>0$ ) and represents the trade-off between the suitability of the data and the estimated model. To check the quality of the inversion, we can use the root mean square error $\left(E_{R M S}\right)$, as follows:

$$
E_{R M S}=\sqrt{\frac{(d-G(m))^{T} W(d-G(m))}{N}} \times 100 \%
$$

where $N$ is the number of data.

\section{RESULTS AND DISCUSSION}

In this study, the initial model is a homogeneous conductivity model of $10-2 \mathrm{~s} / \mathrm{m}$, with the regularization parameter $(\beta)$ being positive in the range $1-1.5$, with 20 iterations.

\section{Inversion Model and True Model}

Figure 2 shows a 2-dimensional conductivity inversion model. Inversion modeling has provided an inversion model for each polarization source, namely TE mode in Figure 2b, TM mode in Figure 2c, and a combination of TE and TM modes in Figure 2d.

In general, the inversion model has a conductivity value of around $10^{-1} \mathrm{~S} / \mathrm{m}$, which is the environmental conductivity, a conductivity of around $10^{-3} \mathrm{~S} / \mathrm{m}$, which is the sediment conductivity and is also overlaid by the conductivity of about $10^{-2} \mathrm{~S} / \mathrm{m}$ which is the surface conductivity, with suitable width and depth of the model. The result shows that the modeling provides significant results where the inversion model fits the actual model.

The model that is the object of observation has a limit point from 15000 to -15000 (x-axis). The shape of the model, which is generated outside the point boundary, is the result of the initial model selected as a whole.

\section{Prediction Data and Observation Data}

In general, the inversion process results in fitting data on the apparent resistivity curve and phase to frequency for each polarization source. For example, the TE mode in Figure 3 and the TM mode 
(a)
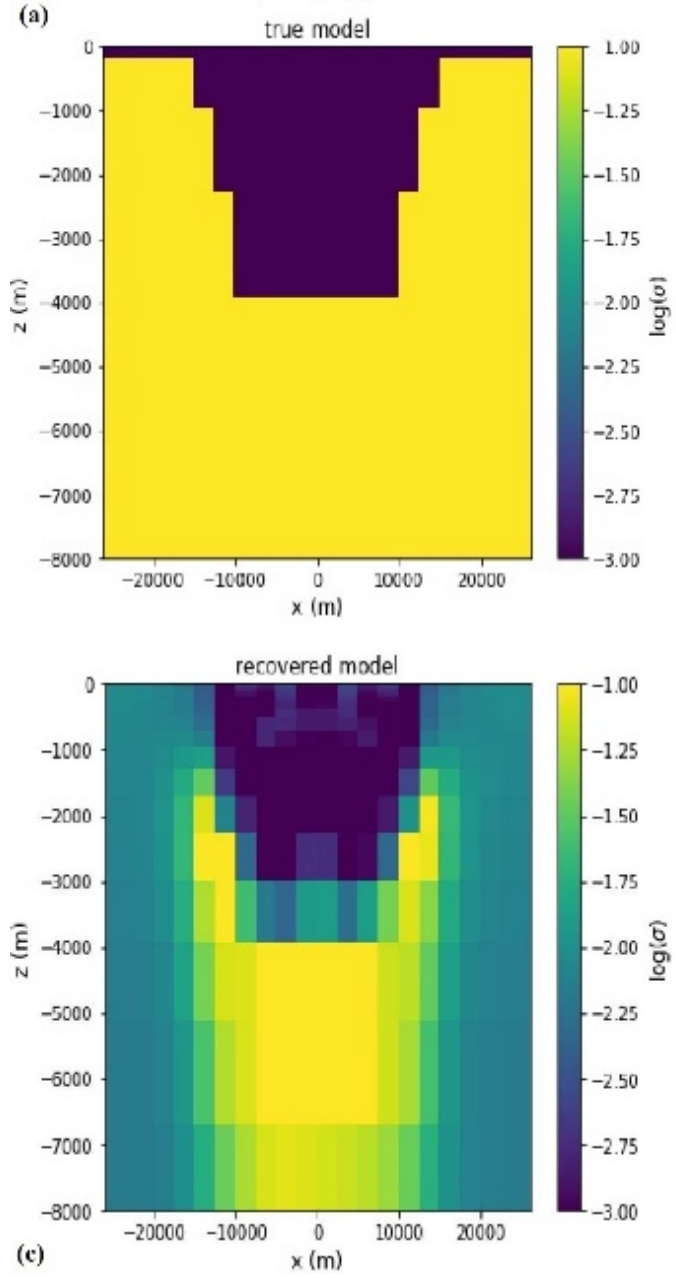

(b)
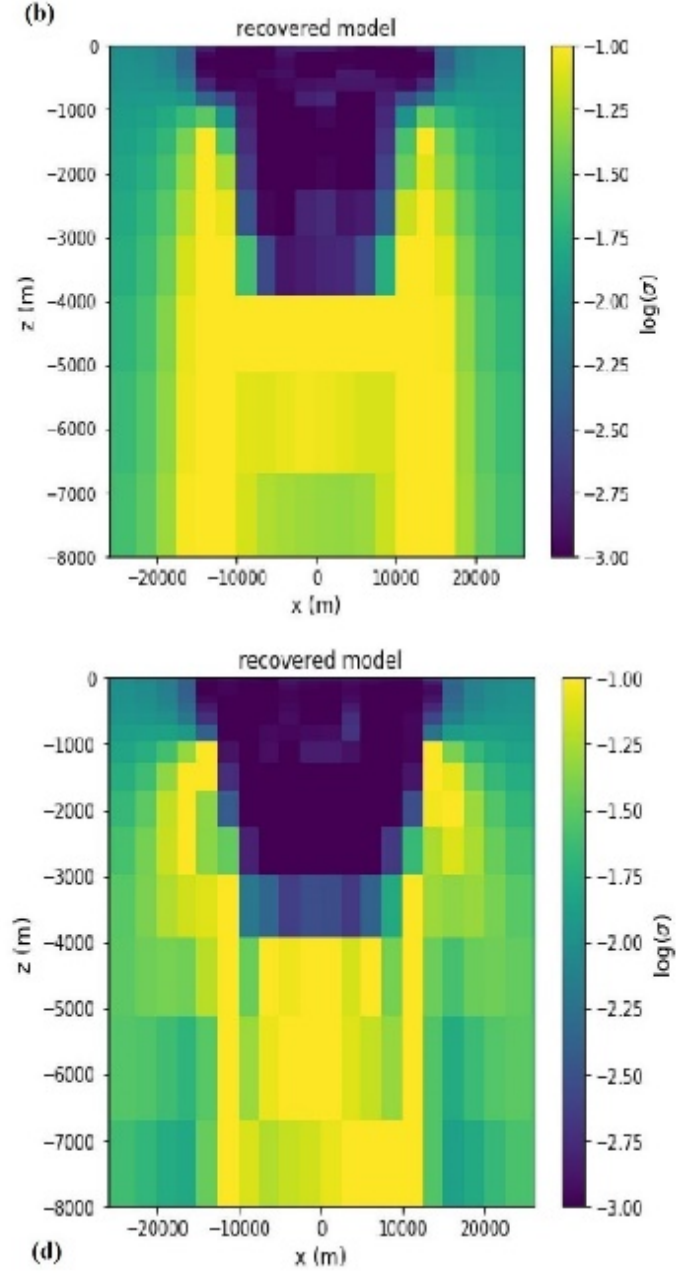

Figure 2. (a) True model, (b) TE mode inversion, (c) TM mode inversion, and (d) joint inversion from TE and TM mode.

in Figure 4 shows a fairly good fit between the prediction data and the observation data at station one $(-12000)$.

The inversion quality can be explained based on the Tikhonov curve, which describes the relationship between the data misfit function (black line) and the model function (red line) on the iteration process. In the process, the iteration will stop if it has reached the target misfit ( $\phi \mathrm{d})$, even though it has not reached the given iteration limit. The misfit target will be achieved if the data misfit function (black line) has reached the limit on the black dotted line and moves away from each other with the model function (red line) in the iteration process. The target misfit can show the quality of data modeling.

In the case of this study, the Tihkonov curve shows the quality of the 2-dimensional inversion modeling process carried out on MT synthetic data for each polarization source, with 20 iterations. In this modeling, TE mode has reached the misfit target in the 11th iteration with an RMS Error value of $0.349 \%$ (see Figure 5.a). TM mode has reached the target of the misfit in the 13th iteration with an RMS Error value of $0.348 \%$ (see Figure 5.b. Meanwhile, the combination of TE and TM modes has reached the misfit target in the 17th iteration with an RMS error of $0.249 \%$ (see Figure 5.c). 

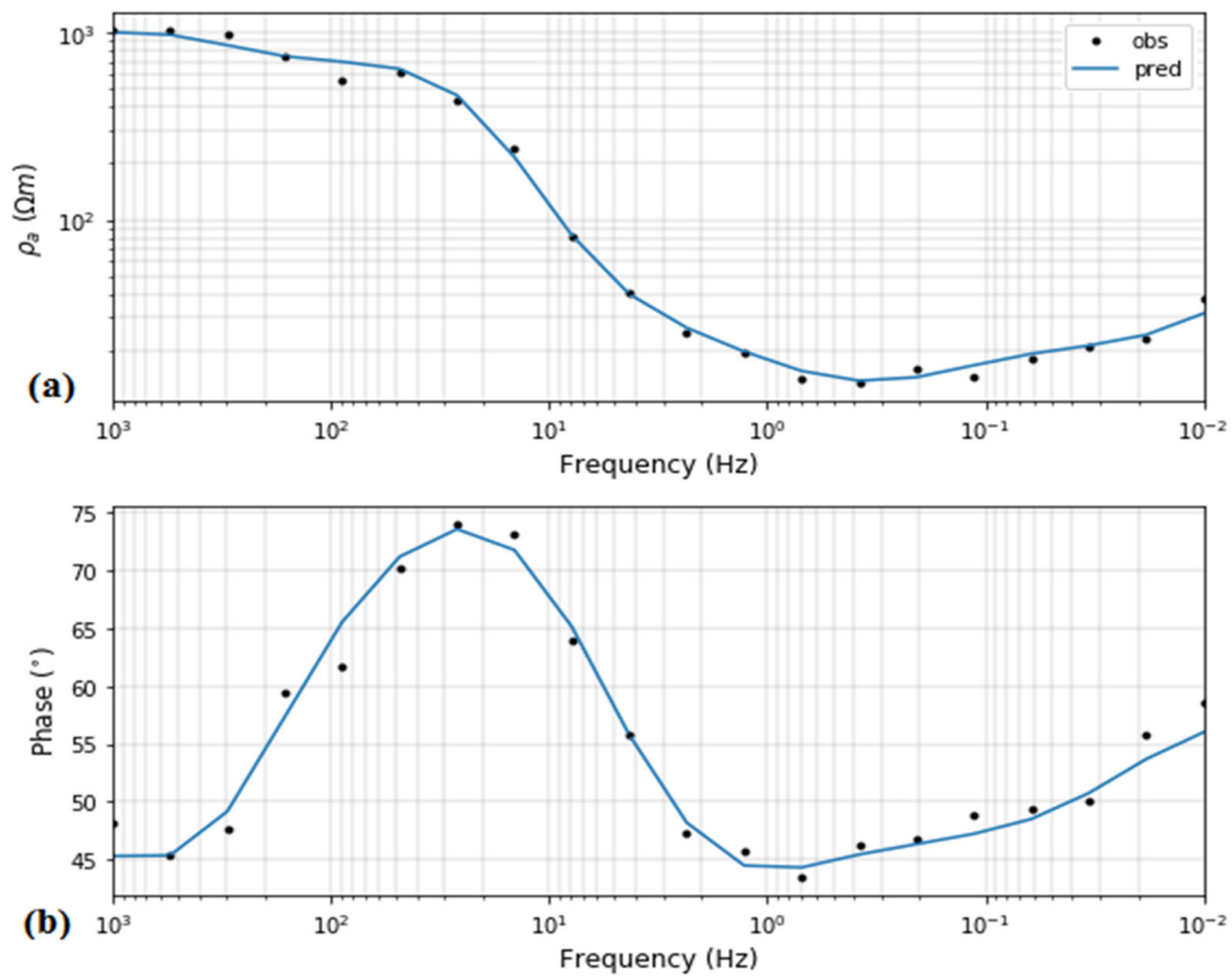

Figure 3. Data Fitting TE mode (a) apparent resistivity, (b) phase to frequency curve at station $1(-12000)$.
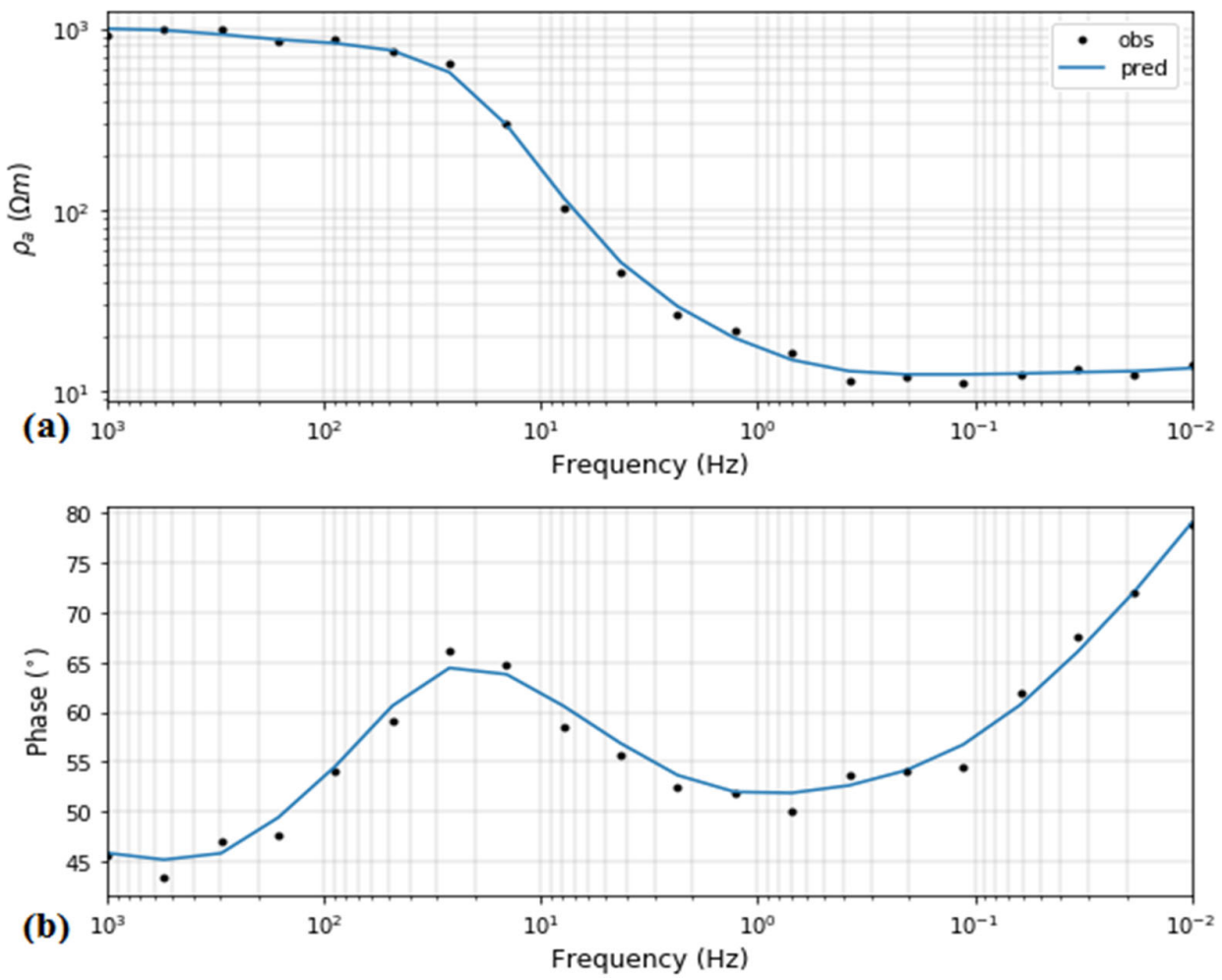

Figure 4. Data Fitting TM mode; (a) apparent resistivity, (b) phase to frequency curve at station $1(-12000)$. 

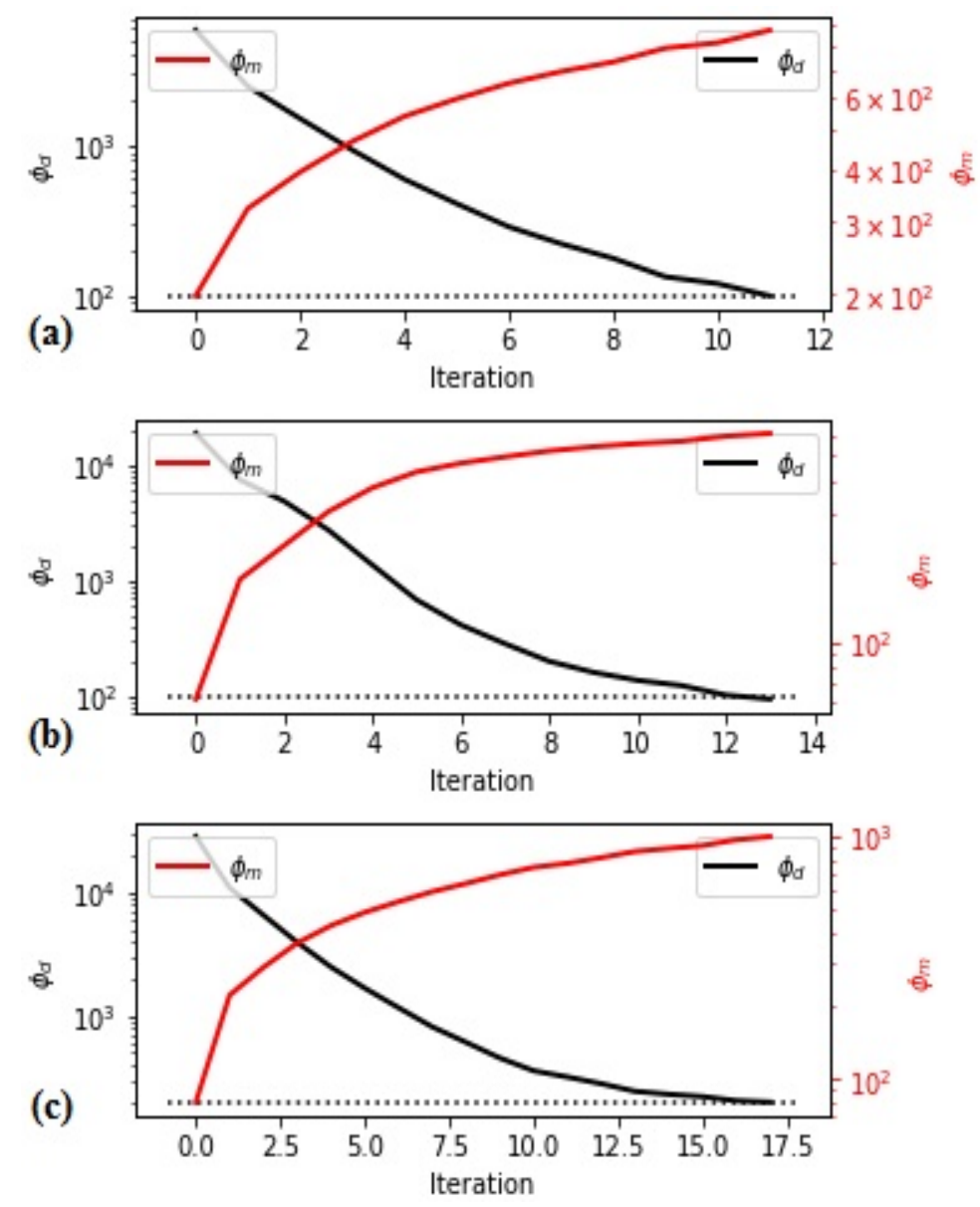

Gambar 5. Tikhonov Curve (a) TE mode, (b) TM mode, and (c) joint $T E$ dan TM mode.

\section{CONCLUSION}

This study concludes that the 2-dimensional inversion modeling carried out on MT synthetic data with a graben morphology model, using five data measurement receivers, with polarization sources, TE mode, TM mode, and a combination of TE and TM modes have resulted in model fit and suitability data at a significant level. The inversion quality has been validated, with RMS Error values of $0.349 \%, 0.348 \%, 0.249 \%$, respectively, for TE, TM, and a combination of TE and TM Modes.

These results indicate that the SimPEG software used for the 2-dimensional inversion modeling process on MT synthetic data involving certain parameters has a fairly high level of validity. It is evidenced by the suitability of the model and the data fitting, which has a minimum error rate. Furthermore, this SimPEG software can be widely applied free of charge for the 2-dimensional inversion modeling process of MT field data.

\section{ACKNOWLEDGMENTS}

The authors would like to thank those who have fully contributed to completing this research and to the Research Center for Geotechnology, the Indonesian Institute of Sciences (LIPI) as the publisher, the anonymous reviewers, and the editorial team of the Journal of Geology and Mining. 


\section{REFERENCES}

Bücker, M., García, S.L., Guerrero, B.O., Caballero, M., Pérez, L., Caballero, L., Pita, C., Paz, D., Sánchez-galindo, A., Villegas, F.J., Orozco, A.F., Brown, E., Werne, J., Garcés, B.V., Schwalb, A., Kemna, A., Sánchez-alvaro, E., Launizar-martínez, N., Valverde-placencia, A., Garay-jiménez, F., 2017. Geoelectrical and Electromagnetic Methods Applied to Paleolimnological Studies : Two Examples from Desiccated Lakes in the Basin of Mexico.

Cagniard, L., 1953. Basic Theory of the Magneto-telluric Method of Geophysical Prospecting. Geophysics 18, 605 - 635.

Chave, A.D., Jones, A.G., 2012. The Magnetotelluric Method Theory and Practice. Cambridge University Press, Cambridge.

Cockett, R., Kang, S., Heagy, L.J., Pidlisecky, A., Oldenburg, D.W., 2015. SimPEG: An Open Source Framework for Simulation and Gradient-Based Parameter Estimation in Geophysical Applications. Comput. Geosci. 85, 142-154. https://doi.org/10.1016/j.cageo.2015.09.015

Grandis, H., 2009. Pengantar Pemodelan Inversi Geofisika. Himpunan Ahli Geofisika Indonesia (HAGI), Bandung.

Heimann, S., Vasyura-bathke, H., Sudhaus, H., Isken, M.P., Kriegerowski, M., Steinberg, A., Dahm, T., 2019. A Python Framework for Efficient Use of Pre-Computed Green's Functions in Seismological and Other Physical Forward and Inverse Source Problems. Solid Earth 10, 1921-1935. https://doi.org/https://doi.org/10.5194/se-10-1921-2019

Key, K., 2016. MARE2DEM: a 2-D Inversion Code for Controlled-Source Electromagnetic and Magnetotelluric Data. Geophys. J. Int. 207, 571-588. https://doi.org/10.1093/gji/ggw290

Loke, M.H., Barker, R.D., 1996. Rapid Least-squares Inversion of Apparent Resistivity Pseudosections by a Quasi-Newton Method. Geophys. Prospect. 44, 131-1 52.

Naidu, G.D., 2012. Magnetotellurics: Basic Theoretical Concepts. Springer-Verlag Berlin Heidelberg. https://doi.org/10.1007/978-3-642-28442-7

Rikitake, T., 1948. Notes on the Electromagnetic Induction within the Earth. Earthq. Res. Inst. 1-9.

Rücker, C., Günther, T., Wagner, F.M., 2017. pyGIMLi : An Open-Source Library for Modelling and Inversion in Geophysics. Comput. Geosci. 109, 106-123. https://doi.org/10.1016/j.cageo.2017.07.011

SimPEG [WWW Document], 2013a. URL https://github.com/SimPEG/tlemagnetotelluric_inversion/blob/master/notebooks/3_MT1D_5layer_inversion.ipynb (accessed 10.17.20).

SimPEG [WWW Document], 2013b. URL https://github.com/SimPEG/SimPEG/blob/master/examples/07nsem/plot_fwd_nsem_MTTipper3D.py (accessed 10.17.20).

Simpson, F., Bahr, K., 2005. Practical Magnetotellurics. Cambridge University Press, Cambridge.

Sism, W.E., Bostick, F.X., 1969. Methods of Magnetotelluric Analysis 1-94.

Tezkan, B., Muttaqien, I., Saraev, A., 2018. Mapping of Buried Faults using the 2D Modelling of Far-field Controlled Source Radiomagnetotelluric Data. Pure Appl. Geophys. 1-16. https://doi.org/10.1007/s00024-018-1980-0

Tikhonov, A.N., 1950. On Determination of Electrical Characteristics of the Deep Layers of the Earth's Crust. Geophys. Inst. Acad. Sci. USSR 2, 295-297.

Uieda, L., Jr, V.C.O., Barbosa, V.C.F., 2013. Modeling the Earth with Fatiando a Terra. Proc. 12th Python Sci. Conf. 92-98.

Unsworth, M., 2009. Introduction to Electromagnetic Exploration Methods. Geophys. 223 1-5.

Unsworth, M., 2007. Magnetotellurics: Encyclopedia of Geomagnetism and Paleomagnetism 1-8.

Varga, M. de la, Schaaf, A., Wellmann, F., 2019. GemPy 1.0: Open-Source Stochastic Geological Modeling and Inversion. Geosci. Model Dev. 12, 1-32. https://doi.org/https://doi.org/10.5194/gmd-12-1-2019

Vozoff, K., Nabighian, M.N., 1991. The Magnetotelluric Method. Chapter 8 In Electromagnetic Methods in Applied Geophysics-Applications Part A and Part B, Volume 2. ed. Society of Exploration Geophysicists, United States of America.

Zonge, K.L., Hughes, L.J., Nabighian, M.N., 1991. Controlled Source Audio-Frequency Magnetotellurics. Chapter 9 In Nabighian, M. N., Electromagnetic Methods in Applied Geophysics Applications Part A and Part B., Volume 2. ed. Society of Exploration Geophysicists, United States of America. 
Muttaqien \& Nurjaman / Two-dimensional inversion modeling of magnetotelluric (MT) synthetic data 